\title{
DRY-RELEASED POST-CMOS COMPATIBLE CONTOUR-MODE ALUMINUM NITRIDE MICROMECHANICAL RESONATORS FOR VHF APPLICATIONS
}

\author{
Gianluca Piazza and Albert P. Pisano
}

\author{
Berkeley Sensor and Actuator Center (BSAC) \\ University of California Berkeley, Berkeley, CA 94720 \\ Tel: (510) 642-8713, Fax: (510) 643-6637,Email: piazza@eecs.berkeley.edu
}

\begin{abstract}
This paper reports experimental results on a new class of dryreleased, post-CMOS compatible, contour-mode aluminum nitride resonators spanning a frequency range from $23 \mathrm{MHz}$ to $225 \mathrm{MHz}$ showing high quality factors both in vacuum $\left(\mathrm{Q}_{\max }=5,830\right.$ at 43.26 $\mathrm{MHz})$ and air $\left(\mathrm{Q}_{\mathrm{air}}=3,700\right)$, and low motional resistance $(\sim 600 \Omega$ at $89 \mathrm{MHz})$. For the first time, thin-film aluminum nitride technology has been used to fabricate circular and rectangular plates, which were successfully excited in contour modes in both fundamental and higher order modes. Dilation-type modes were excited in AlN rectangular plates demonstrating frequencies as high as $224.6 \mathrm{MHz}$ with Q of 2,580. A $50 \mu \mathrm{m}$ radius disk vibrating in a wineglass contour mode exhibited Q of 5,830 at $43.26 \mathrm{MHz}$. Uncompensated temperature coefficients of frequency were measured to be $\sim-14 \mathrm{ppm} /{ }^{\circ} \mathrm{C}$ for the disk and $\sim-22 \mathrm{ppm} /{ }^{\circ} \mathrm{C}$ for a $200 \times 50 \mu \mathrm{m}^{2}$ rectangular plate vibrating in its $3^{\text {rd }}$ mode.
\end{abstract}

\section{INTRODUCTION}

Recent demand in wireless communication for miniaturized, low-power, low-cost, on-chip and high-Q resonators to be employed in front-end RF filters or as frequency references has focused research efforts towards the development of new vibrating micromechanical structures, capable of substituting existing offchip, bulky resonator technologies. Very promising alternatives to currently adopted solutions (SAW or ceramic devices) have been demonstrated [1,2] using in-plane, electrostatically-transduced, micromechanical resonators made of polysilicon or polydiamond. Although extremely high quality factors have been reported at UHF, the exhibited impedance values are too high for these resonators to be directly coupled to antennas in RF systems. Also, the high temperature fabrication steps involved with the deposition of the structural layers ultimately complicate the integration of these devices with state-of-the-art microelectronic components.

FBAR technology $[3,4]$ has proven itself as a valid solution to replace conventional RF filters, demonstrating relatively high quality factors $(\mathrm{Q} \sim 500)$, and extremely small (several $\Omega$ ) impedances. The fundamental frequency of these devices is set by the film thickness. This constitutes a major challenge to the manufacturing of FBARs. In order to obtain reasonable yields, a thickness tolerance of $0.1 \%$ must be met. Also, multiple frequency selective arrays of resonators cannot be fabricated on a single chip. The new class of devices described here is not subject to these limitations.

This work introduces a new class of aluminum nitride micromechanical resonators that exhibit high quality factors, small impedance values $(\sim 600 \Omega$ at $89 \mathrm{MHz})$ and are fabricated using a low-temperature, post-CMOS compatible surface micromachining process. For the first time, thin film aluminum nitride technology has been used to fabricate circular and rectangular plates, which were successfully excited in contour modes in both fundamental and higher order modes. The use of contour modes specifically enables the fabrication of arrays of piezoelectric microresonators with different frequencies on a single chip. Other piezo-on-silicon rectangular plates have been described in the literature [5], however these resonators did not employ the same contour modes. The research described in this paper has led to resonators with low insertion losses, demonstrating that a fully aluminum nitride resonator body can effectively reduce the equivalent motional resistance and simultaneously achieve high quality factors. Most importantly these devices can be operated in air without significant $\mathrm{Q}$ degradation $\left(\mathrm{Q}_{\text {vacuum }}=3,280\right.$ and $\mathrm{Q}_{\mathrm{air}}=2,680$ for a $200 \times 50 \mu \mathrm{m}^{2}$ plate). Low, uncompensated temperature coefficients of frequency (TCF) of $\sim-14 \mathrm{ppm} /{ }^{\circ} \mathrm{C}$ for disks and $\sim-22 \mathrm{ppm} /{ }^{\circ} \mathrm{C}$ for a $200 \times 50 \mu \mathrm{m}^{2}$ plate ( $\sim 2 \mathrm{X}$ smaller than reported in [5]) have also been demonstrated. In addition, the center-frequency has been tuned both up and down by purely piezoelectric means. A tuning range of $\sim 6 \mathrm{kHz}$ was obtained for a $22.97 \mathrm{MHz}$ rectangular plate using a 30V DC power supply.

\section{CONTOUR MODES IN AIN PLATES}

Figures $1 \mathrm{a}$ and $1 \mathrm{~b}$ show schematic views of the AlN rectangular plates vibrating in dilation-type contour modes. A vertical electric field applied across the film thickness induces extensional mechanical stress in the plane of the film (through the $\mathrm{d}_{31}$ piezoelectric coefficient) and excites the structures in a dilation-type contour mode.

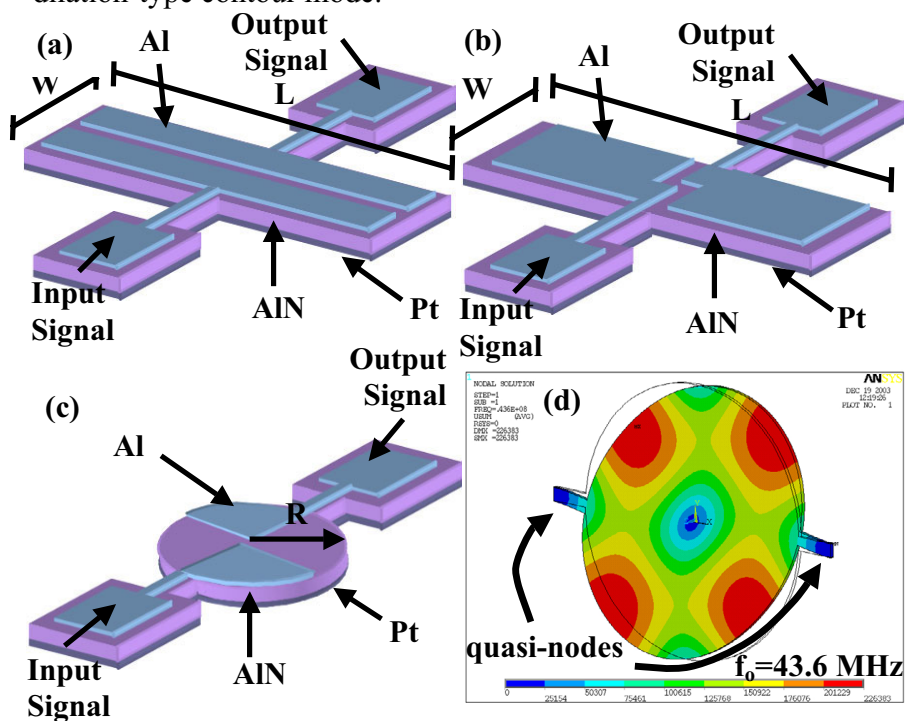

Figure 1. Two-port AlN micromechanical resonators: (a) schematic view of an AlN rectangular plate with electrodes placed parallel to the plate length; (b) schematic view of an AlN rectangular plate resonators with electrodes placed parallel to the plate width; (c) schematic view of an AlN circular plate excited in a wineglass contour mode shape (d). Mode shapes for (a) and (b) are shown in Fig.2. 

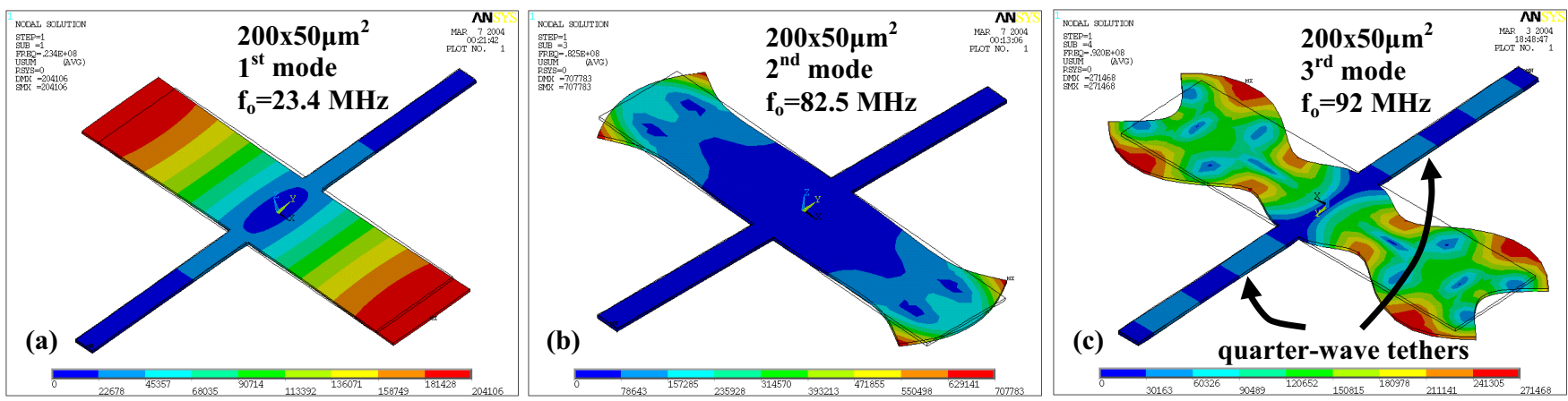

Figure 2. Dilation-type contour modes (via FEA) excited in two-port AlN rectangular plate(200x50 um $^{2}$ ) resonator: (a) fundamental length-extensional mode shape (denoted as $1^{\text {st }}$ mode in this paper); (b) $1^{\text {st }}$ width-extensional mode shape (denoted as $2^{\text {nd }}$ mode in this paper); (c) $2^{\text {nd }}$ width-extensional mode showing effective "quarter-wavelength" tethers design (denoted as $3^{\text {rd }}$ mode in this paper).

Two-port configurations were implemented by patterning the top electrodes symmetrically with respect to the length or the width of the plate. In order to minimize anchor losses, the resonators were suspended by two "quarter-wavelength" tethers.

Several in-plane contour mode shapes can be excited in these resonators [6], but either electrode configurations or energy loss mechanisms limit the detectable mode shapes to the one shown in Fig.2. The resonator can most effectively be excited in lengthextensional (Fig. 2a) [7] and width-extensional (Fig. 2b-c) dilationtype contour modes. An important parameter that characterizes the relative strength of each of the resonances and sets the value of the equivalent motional resistance is the electromechanical coupling, $\eta_{\mathrm{em}}$. It can either be defined as the ratio of the output force to the input voltage, or the ratio of the output charge to the mechanical displacement. For a symmetric electrode topology such as the one used in this work, $\eta_{\text {in }}$ and $\eta_{\text {out }}$ are the same; specifically:

$$
\eta_{e m}=\frac{\iint_{A}\left(d_{31} T_{1}+d_{31} T_{2}\right) d A}{Z_{\max }}=\frac{\frac{2 E}{1-\sigma} d_{31} \iint_{A}\left(\frac{\partial u}{\partial x}+\frac{\partial v}{\partial y}\right) d A}{Z_{\max }}
$$

where $T_{1}$ and $T_{2}$ represent normal stresses in the $x$ and $y$ directions respectively, $d_{31}$ is the piezoelectric coefficient, $u$ and $v$ are the displacements in $x$ and $y$ directions [8], $E$ is the Young's modulus, and $\sigma$ the Poisson ratio for AlN, $A$ is the electrode area, and $Z_{\max }$ is the maximum displacement for the structure. Taking this into account the electrodes were placed so that $\eta_{\mathrm{em}}$ is maximized.

\section{WINEGLASS MODE IN AIN DISKS}

In Fig.1c is shown a schematic view of an AlN micromechanical disk to be excited in a wineglass contour mode. Thanks to in plane symmetry of AlN films, radial and compound mode shapes [9] can be effectively excited in thin circular disks through the $d_{31}$ piezoelectric coefficient. In order to minimize anchor losses, the disk was pinned at two of its quasi-nodal points (Fig.1d).

A two-port configuration is implemented by having each of the two top electrodes cover a quadrant of the disk. By placing the electrode in such a fashion, maximum electromechanical coupling is achieved. In this specific case $\eta_{\mathrm{em}}$ is given by:

$$
\eta_{e m}=\frac{\frac{2 E}{\left(1-\sigma^{2}\right)} d_{31} \iint_{A}\left[\frac{\partial u}{\partial r}+\sigma\left(\frac{u}{r}+\frac{1}{r} \frac{\partial v}{\partial r}\right)\right] d A}{Z_{\max }}
$$

where $E, \sigma, A$ and $Z_{\max }$ are the same as defined in equation (1), $u$ and $v$ represent the radial and tangential displacements [9], respectively, and $r$ is the radial coordinate for the circular plate. Due to the isovoluminal nature of the mode shape and the electrode covering just half of the plate top surface, the electromechanical coupling for the disk is relatively smaller $(\sim 8 \mathrm{X})$ than that for AlN plates of comparable frequency.

\section{FABRICATION PROCESS}

A simple three-mask, low-temperature $\left(\mathrm{T}_{\max }<350^{\circ} \mathrm{C}\right)$, postCMOS compatible process has been used to fabricate rectangular and circular AlN plates (Fig. 3). A thin ( $\sim 50 \mathrm{~nm})$ AlN buffer layer is deposited on a $\mathrm{Si}$ wafer to provide electrical isolation. A $1.5 \mu \mathrm{m}$ AlN film is sandwiched between a bottom platinum electrode and a top aluminum electrode. AIN is reactively deposited using an Advanced Modular Sputtering Inc., AMS 2003 sputtering tool. $\mathrm{Cl}_{2}$-based dry etching has been used to pattern AlN and yields fairly straight (within $16^{\circ}$ of vertical) sidewalls (Fig.5) with an etch rate of $\sim 80 \mathrm{~nm} / \mathrm{min}$. Oxide deposited by electron cyclotron resonance is used as a hard mask during the AlN etching step and the ion milling of the Pt layer. Openings to contact the bottom electrode are quickly wet etched through AlN in a hot $\left(160^{\circ} \mathrm{C}\right)$ phosphoric bath. The top $\mathrm{Al}$ electrode is evaporated and strategically patterned so that maximum electromechanical coupling is obtained. The structures are released by dry etching of silicon in $\mathrm{XeF}_{2}$. This novel dry release step for fully AIN resonators eliminates stiction forces and significantly increases yield compared to other processes that use wet release techniques [5].

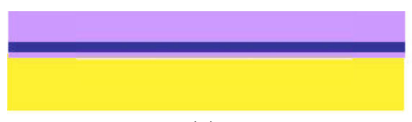

(a)

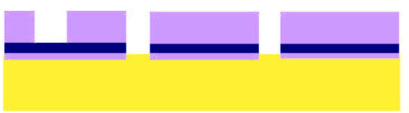

(c)

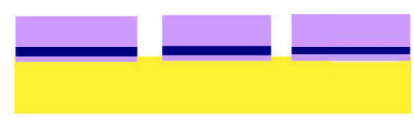

(b)

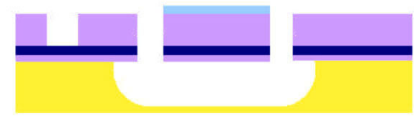

(d)
Si $\square$ Pt $\square$ AIN $\quad$ Al

Figure 3. Fabrication process flow for AlN micromechanical resonators: (a) AlN,Pt and AlN are sputter-deposit on a Si wafer; (b) trenches are open through $\mathrm{AlN}$ and $\mathrm{Pt}$ by $\mathrm{Cl}_{2}$-based dry etching and ion milling, respectively; (c) Openings to the bottom electrode are wet etched through AlN in hot $\left(160^{\circ} \mathrm{C}\right) \mathrm{H}_{3} \mathrm{PO}_{4}$; (d) Al top electrodes are evaporated and patterned by lift-off. 

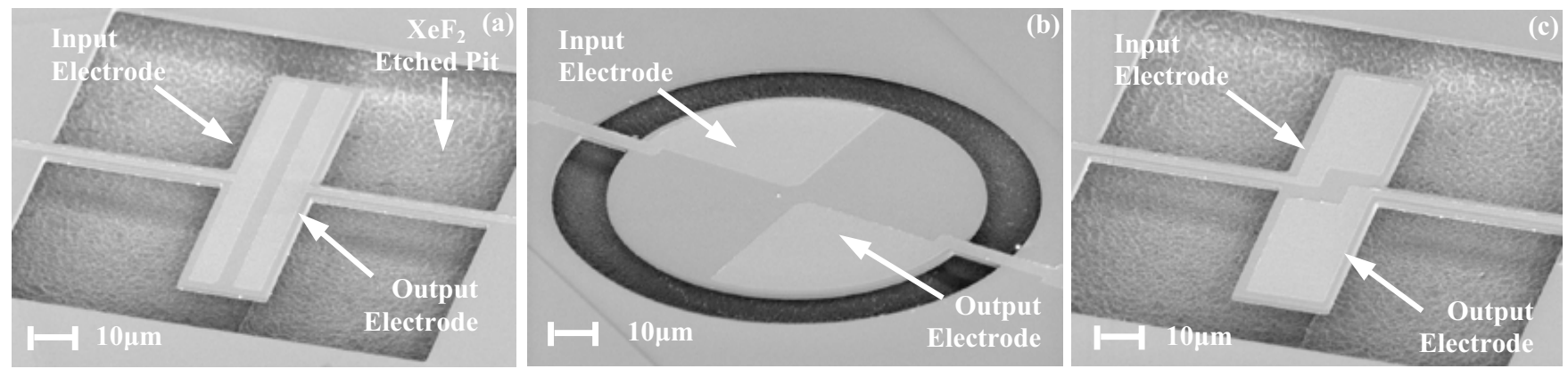

Figure 4. Scanning electron microscope pictures showing: (a) $80 \times 20 \mu \mathrm{m}^{2}$ AlN rectangular plate with electrodes parallel to the resonator length; (b) $50 \mu \mathrm{m}$ radius AlN circular plate to be excited in a wineglass mode shape; (c) $80 \times 20 \mu \mathrm{m}^{2}$ AlN rectangular plate with electrodes parallel to the resonator width.
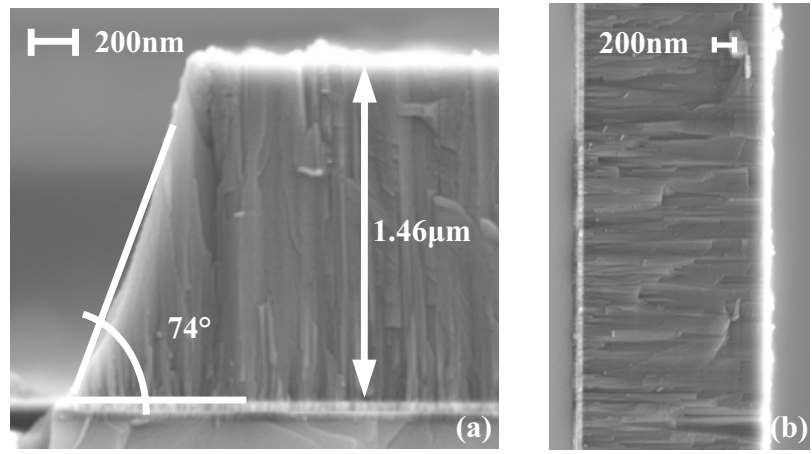

Figure 5. Cross-section views of AlN films: (a) $74^{\circ}$ sidewalls are obtained after $\mathrm{Cl}_{2}$ dry etch and ion milling; (b) closely-packed, highly crystalline AlN film is obtained using AMS sputtering tool (rocking curve value: best $F W H M \sim 1.9^{\circ}$ ).

All the deposition steps occur at low temperature $\left(\mathrm{T}_{\max }\right.$ $\sim 350^{\circ} \mathrm{C}$ when sputtering AlN) and can potentially be integrated with state-of-the-art microelectronic components. Fig. 4 shows rectangular and circular plates that were fabricated using the aforementioned process.

\section{EXPERIMENTAL RESULTS}

The fabricated micromechanical resonators were tested in a Janis micro-manipulated RF vacuum probe system in 5mTorr vacuum and at atmospheric pressure. Ground-Signal-Ground (GSG) probes from Picoprobe were used. The bottom Pt electrode was grounded, whereas each of the two top electrodes was used for either sensing or driving the device under test. No interface circuitry between the resonator and the network analyzer was needed. Given the low impedance values, it was possible to directly measure the frequency response of the resonators using an Agilent 4195A network analyzer.

\section{A. Frequency Response of AIN Rectangular Plates}

Various rectangular plates with length to width ratios of 2 and 4 were fabricated and tested. The resonators varied in length from 80 to $200 \mu \mathrm{m}$. All resonators, despite their size and electrode configuration, exhibited the fundamental length-extensional mode. Fig. 6a represents the typical response in vacuum of a $200 \times 50 \mu \mathrm{m}^{2}$ plate, showing $\mathrm{Q}$ of 3,280 and a motional resistance, $\mathrm{R}_{\mathrm{x}}$, of only $\sim 150 \Omega$ when vibrating in its $1^{\text {st }}$ HF mode (Fig.2a). It was also possible to excite higher order modes in plates with electrodes placed parallel to the plate length and having a length to width ratio of 4 . $\mathrm{Q}$ as high as 4,470 were obtained from the $2^{\text {nd }}$ mode
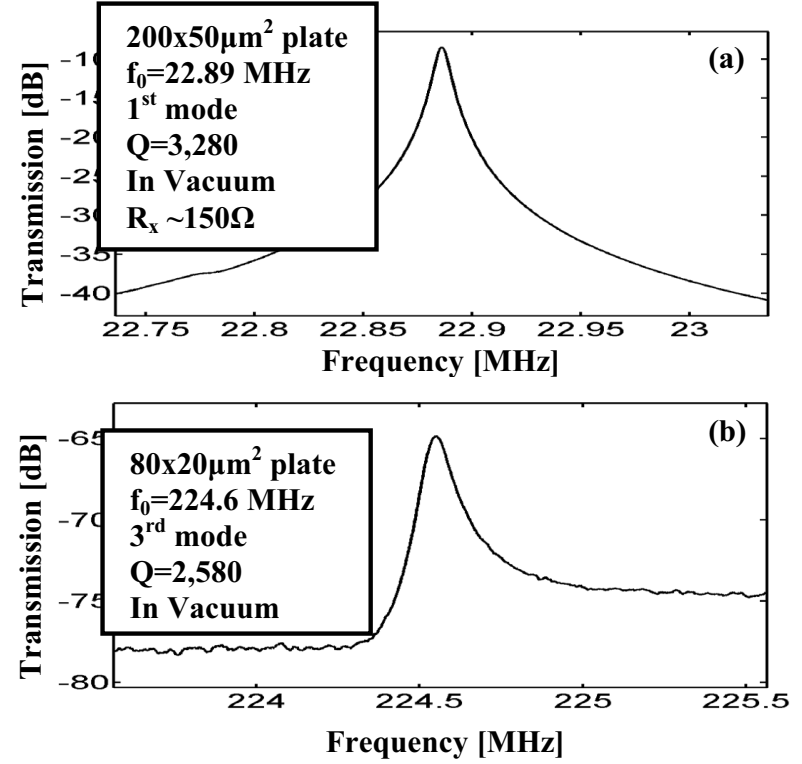

Figure 6. (a) Typical frequency response of a $200 \times 50 \mu^{2}$ rectangular plate excited in its fundamental mode in vacuum; (b) frequency response for a $80 \times 20 \mu^{2}$ rectangular plate excited in its $3^{\text {rd }}$ mode in vacuum.

(Fig.2b) of a $200 \times 50 \mu \mathrm{m}^{2}$ plate at $80.57 \mathrm{MHz}$ in vacuum. A $80 \mathrm{x} 20 \mu \mathrm{m}^{2}$ plate achieved the highest measured frequency of 224.6 $\mathrm{MHz}$ with Q of 2,580 (Fig. 6b) in vacuum, when vibrating in its $3^{\text {rd }}$ mode (Fig. 2c). The use of quarter-wavelength tethers minimized the energy lost through the anchors and enabled achieving quality factors higher than the one recorded for the $1^{\text {st }}$ mode. The same plate could not be excited in its $2^{\text {nd }}$ mode. The low electromechanical coupling exhibited by this mode shape is most likely why it cannot be detected at higher frequencies. It is interesting to note that these resonators could be actuated in air without significant $\mathrm{Q}$ degradation. Recorded $\mathrm{Q}$ reduction was about $20-30 \%$ for all resonators. For example, Q of 2,000 in air and $\mathrm{Q}$ of 2,580 in vacuum were recorded for a $80 \times 20 \mu \mathrm{m}^{2}$ plate vibrating in its $3^{\text {rd }}$ mode. Experimental measurements for dilationtype contour modes in AlN rectangular plates are summarized in Table I. Recorded frequencies match within 3\% to ANSYS finite element analysis (electrode loading was not taken into account for FEA).

A $200 \times 50 \mu \mathrm{m}^{2}$ resonant plate exhibited a linear TCF over a temperature range of $28-100^{\circ} \mathrm{C}$. TCF values of $\sim-26 \mathrm{ppm} /{ }^{\circ} \mathrm{C}$, $\sim-25 \mathrm{ppm} /{ }^{\circ} \mathrm{C}$, and $\sim-22 \mathrm{ppm} /{ }^{\circ} \mathrm{C}$ were recorded for the same plate vibrating in its $1^{\text {st }}, 2^{\text {nd }}$ and $3^{\text {rd }}$ mode, respectively. Furthermore it was possible to tune the center-frequency of a plate both up and 
down by purely piezoelectric means. A constant strain was induced in the resonator by superimposing a DC voltage to the ac signal on the two top electrodes. This tuning mechanism resulted in a $\pm 3 \mathrm{kHz}$ linear tuning range for a $22.97 \mathrm{MHz}$ rectangular plate using a $30 \mathrm{~V}$ power supply (slope $\sim 4.4 \mathrm{ppm} / \mathrm{V}$ ). Such a tuning scheme could potentially be employed to implement low-power active frequency compensation for temperature variations of $\pm 10^{\circ} \mathrm{C}$.

Table I. Summary of most significant experimental measurements for dilation-type contour modes in AlN rectangular plate. $A$ and $B$ next to the plate size refer to the top electrode configuration: $A$ indicates resonators with electrodes parallel to the length of the plate, $B$ indicates resonators with the electrodes parallel to the width of the plate. I.L. stands for insertion loss. Modes are numbered as defined in Fig.2.

\begin{tabular}{|c|c|c|c|c|}
\hline $\begin{array}{c}\text { Size } \\
\text { LxW [MHz] }\end{array}$ & Mode & $\begin{array}{c}\mathbf{F}_{\mathbf{O}} \\
{[\mathbf{M H z}]}\end{array}$ & $\begin{array}{c}\text { Q } \\
\text { (in vacuum) }\end{array}$ & $\begin{array}{c}\text { I.L. } \\
{[\mathbf{d B}]}\end{array}$ \\
\hline 200x100 A & 1 & 22.88 & 2,820 & -16.7 \\
\hline $200 \times 50 \mathrm{~A}$ & 1 & 22.89 & 3,280 & -9 \\
\cline { 2 - 5 } & 2 & 80.57 & 4,470 & -23.1 \\
\hline & 3 & 88.99 & 4,200 & -21.8 \\
\hline 140x70 A & 1 & 32.66 & 1,360 & -32.2 \\
\hline $140 \times 35$ A & 1 & 32.65 & 1,860 & -29.4 \\
\hline & 2 & 115.3 & 2,870 & -32.9 \\
\hline & 3 & 127.3 & 1,520 & -36.4 \\
\hline $140 \times 35$ B & 1 & 32.72 & 3,320 & -32.2 \\
\hline $80 \times 20$ A & 1 & 57.67 & 1,940 & -40.6 \\
\hline & 3 & 224.6 & 2,580 & -64.8 \\
\hline $80 \times 20$ B & 1 & 57.40 & 1,885 & -39.1 \\
\hline
\end{tabular}

\section{B. Frequency Response of AIN Disks}

$50 \mu \mathrm{m}$ radius AlN disks vibrating in a wineglass contour mode shape exhibited Q as high as 5,830 at a frequency of $43.26 \mathrm{MHz}$ in vacuum (Fig. 7). A high quality factor of 3,700 was recorded in air for the same type of resonators. Using fairly small tethers $(5 \mu \mathrm{m}$ wide) and anchoring the disk at its quasi-nodal points (tangential displacement is non-zero at this locations) resulted in the highest $\mathrm{Q}$ for contour modes in AIN plates. Despite its high Q the resonator shows a motional resistance $(\sim 73 \mathrm{k} \Omega)$ much higher than the one recorded for rectangular plates. Although, having the electrodes over the whole resonator surface could decrease the motional resistance by a factor of 4 , the equivoluminal nature of the mode shape makes its excitation very difficult in AlN films. The same resonator exhibited a linear TCF of $\sim-14 \mathrm{ppm} /{ }^{\circ} \mathrm{C}$ for a temperature range of $28-100^{\circ} \mathrm{C}$. The smaller value of TCF

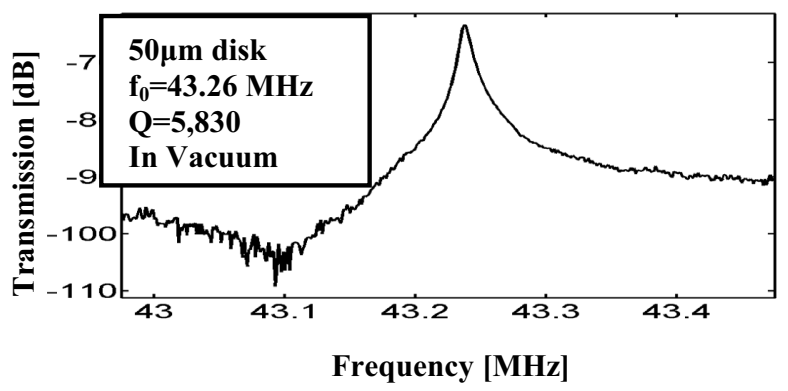

Figure 7. Characteristic frequency response of $50 \mu \mathrm{m}$ radius AlN disk excited in a wineglass contour mode, showing $Q$ of 5,830 at 43.26 MHz. Antiresonance peak appears before series resonance peak because the output voltage is $180^{\circ}$ out of phase with respect to the input voltage [10]. compared to the one recorded for rectangular plates is probably a consequence of the isovoluminal mode shape.

\section{CONCLUSIONS}

For the first time contour-modes were excited in thin-film AlN micromechanical resonators spanning a frequency range from 23 to $225 \mathrm{MHz}$. Rectangular and circular plates were fabricated using a dry-release surface micromachining process that is potentially post-CMOS compatible. Thanks to the use of a fully AlN resonator body and an intrinsically high electromechanical coupling, rectangular plates exhibited impedance values as low as $\sim 150 \Omega$. Three fundamental contour modes were observed in thin rectangular plates, showing Q as high as 4,470 at $80.57 \mathrm{MHz}$. Quarter-wavelength supports enabled achieving high frequencies and high $\mathrm{Q}$ at the same time. Compared to commercially available piezoceramics used for VHF applications, these rectangular plate resonators offer key advantages in terms of size reduction and possible integration with state-of-the-art microelectronic, while maintaining the same quality factors. Contour-mode shapes were also uniquely excited in circular plates. The highest $\mathrm{Q}$ of 5,830 was recorded for a $50 \mu \mathrm{m}$ radius disk excited in a wineglass mode. Ongoing research efforts focus on extending this promising technology to $\mathrm{GHz}$ frequencies.

\section{ACKNOWLEDGMENT}

The work was supported by DARPA grant No. NBCH1020005. The authors wish to thank AMS Inc. for depositing AIN films. Special thanks to UCB microfabrication laboratory staff, Phil Stephanou and Brian Bircumshaw for their help. Travel support has been generously provided by the Transducers Research Foundation and by the DARPA MEMS and DARPA BioFlips programs.

\section{REFERENCES}

1. S.-S. Li, et al., "Micromechanical "Hollow-Disk" Ring Resonators", IEEE MEMS 2004, January 2004, pp. 821-824.

2. J. Wang, et al, "1.51-GHz Nanocrystalline Diamond Micromechanical Disk Resonator with Material-Mismatched Isolating Support", IEEE MEMS 2004, January 2004, pp. 641-644.

3. R. Aigner, et al., " RF-Filters in Mobile Phone Applications", Transducers'03, June 8-12, 2003, pp.891-894

4. R. Ruby, et al., "Ultra-Miniature High-Q Filters and Duplexers Using FBAR Technology", IEEE International Solid-State Circuits Conference, Feb. 2001, pp.121-122.

5. S. Humad, et al., "High Frequency Micromechanical Piezo-OnSilicon Block Resonators", IEDM, 2003, pp. 957-960.

6. R. Holland, "Contour Extensional Resonant Properties of Rectangular Piezoelectric Plates", IEEE Transaction on Sonics and Ultrasonics, Vol. Su-15, No.2, April 1968, pp. 97-105.

7. B. Antkowiak, et al., "Design of High-Q, Low-Impedance, GHz-Range Piezoelectric MEMS Resonators”, Transducers' 03, June 8-12, 2003, pp. 841-846.

8. R.A. Johnson, Mechanical Filters in Electronics, New York, NY Wiley, 1983.

9. M. Onoe, "Contour Vibrations of Isotropic Circular Plates", The Journal of the Acoustical Society of America, Vol. 28, No. 6, pp. 1158-1162.

10. G.Piazza, et al., "Voltage-Tunable PiezoelectricallyTransduced Single-Crystal Silicon Micromechanical Resonators on SOI Wafer", IEEE MEMS 2003, January 2003, pp.149-152. 